\title{
GELATIO: a general framework for modular digital analysis of high-purity Ge detector signals
}

\author{
M. Agostini, ${ }^{a}$ L. Pandola, ${ }^{b}$ P. Zavarise ${ }^{b, c}$ and O. Volynets ${ }^{d}$ \\ a Physik-Department E15, Technische Universität München, \\ James-Franck-Str. 1, D-85748 Garching, Germany. \\ ${ }^{b}$ INFN Laboratori Nazionali del Gran Sasso, \\ SS 17-bis km 18+910, I-67100, Assergi (AQ), Italy. \\ ${ }^{c}$ Dipartimento di Fisica, Università dell'Aquila, \\ Via Vetoio Località Coppito, I-67100, L'Aquila, Italy. \\ ${ }^{d}$ Max-Planck-Institut für Physik, \\ Föhringer Ring 6, D-80805, München, Germany. \\ E-mail: matteo.agostinieph.tum.de, luciano.pandola@lngs.infn.it, \\ paolo.zavarisedlngs.infn.it, volynets emppmu.mpg.de
}

\begin{abstract}
GELATIO is a new software framework for advanced data analysis and digital signal processing developed for the GERDA neutrinoless double beta decay experiment. The framework is tailored to handle the full analysis flow of signals recorded by high purity Ge detectors and photo-multipliers from the veto counters. It is designed to support a multi-channel modular and flexible analysis, widely customizable by the user either via human-readable initialization files or via a graphical interface. The framework organizes the data into a multi-level structure, from the raw data up to the condensed analysis parameters, and includes tools and utilities to handle the data stream between the different levels. Gelatio is implemented in C++. It relies upon Root and its extension TAM, which provides compatibility with PROOF, enabling the software to run in parallel on clusters of computers or many-core machines. It was tested on different platforms and benchmarked in several GERDA-related applications. A stable version is presently available for the GERDA Collaboration and it is used to provide the reference analysis of the experiment data.
\end{abstract}

KEYWORDS: Software architectures (event data models, frameworks and databases), Data processing methods, Gamma detectors (scintillators, CZT, HPG, HgI etc). 


\section{Contents}

1. Introduction 目

2. Concept and design 20

2.1 Multi-level data structure

2.2 Modular digital signal processing

3. Implementation

3.1 Conversion of raw data to the analysis format

3.2 Implementation of digital signal processing

3.3 Utilities 8

3.4 The graphical interface 8

4. Application and Benchmarking 10

5. Conclusions 11

\section{Introduction}

The "GERmanium Detector Array" (GERDA) is an experiment looking for neutrinoless double beta decay of ${ }^{76} \mathrm{Ge}$ which is presently under commissioning at the underground Laboratori Nazionali del Gran Sasso of INFN, Italy [1], 2]1. The neutrinoless double beta decay is a process which violates by two units the lepton number conservation and is forbidden in the Standard Model. Its experimental observation would imply the Majorana nature of the neutrino, also providing a first measurement of the neutrino absolute mass scale. The experiment will use an array of high-purity germanium (HPGe) detectors isotopically enriched in ${ }^{76} \mathrm{Ge}$ (about $40 \mathrm{~kg}$ are planned for the final configuration), aiming to achieve a substantial reduction in the background at the $Q_{\beta \beta}$-value of the ${ }^{76} \mathrm{Ge}$ decay with respect to the predecessor experiments [3, [, []].

The background reduction in GERDA is obtained by an innovative design approach in which naked HPGe detectors are operated directly in ultra radio-pure liquid argon which acts as coolant material and as passive shielding against the external radiation. The cryogenic liquid is surrounded by an additional thick layer of ultra-pure water, which is effective in shielding external neutrons and $\gamma$-rays. The water volume is instrumented with photo-multipliers and is operated as a Cherenkov detector to reject events due to high-energy muons. Part of the remaining background events can be identified by analyzing the HPGe signal shapes and applying pulse shape discrimination (PSD) techniques [ [6, Z, 8, Q]. Moreover, the GERDA collaboration is testing in the R\&D set-up called LARGE [10] the liquid argon instrumentation with photo-multipliers (PMTs) which would provide an active veto system. 
In the present commissioning phase, an array of three non-enriched HPGe detectors has been deployed in the GERDA set-up and is in data taking. The charge signals from the HPGe detectors are sampled by 14-bit Flash-ADCs (FADC) running at $100 \mathrm{MHz}$ sampling rate and stored to disk for off-line analysis. For each physical trigger all the HPGe detector signals are acquired to check for coincidences. A second data stream of the experiment is provided by the PMT signals from the Cherenkov muon veto, which are digitized by the same FADCs used for the HPGe detectors.

In this paper a data analysis framework called GELATIO (GErda LAyouT for Input/Output) is presented and discussed. The framework was developed to handle the full data analysis flow of GERDA as well as of all the R\&D activities related to the experiment. The framework has been designed to be solid, user-friendly, flexible, maintainable over a long lifetime and scalable to the future phases of the experiment. Furthermore, thanks to its generic interfaces, it could be used in other activities involving off-line analysis of digitized pulses from HPGe detectors or other kinds of detectors.

The paper is organized as follows. In section 2 the main requirements driving the software design and the basic concepts of the framework are presented in detail. Section 3 describes the software implementation and the technical solutions pursued. A few examples about the validation and the application of the framework are reported in section 4 . A summary and discussion of future plans are eventually presented in the final section.

\section{Concept and design}

Gelatio is a data analysis framework designed to provide a flexible environment and a complete suite of tools for off-line digital signal processing and for analysis of data recorded with HPGe detectors. The software aims to provide a common platform for the GERDA Collaboration to run the analysis of the experiment data. To meet these requirements the framework must be able to:

- decouple the algorithm implementation from the raw data format allowing the users to run the same analysis algorithms on data sets acquired with different hardware and / or encoded in different formats;

- perform a modular and highly customizable digital signal processing. This approach aims to simplify and foster as much as possible the re-use, the sharing and the comparison of the analysis algorithms, avoiding unnecessary duplications in the code and improving its validation;

- optimize the computational performances and be cross-platform compatible, hiding the technical aspects to the end users.

The solution worked out is based on two paradigms which are discussed below: multiple level data organization and modular digital signal processing.

\subsection{Multi-level data structure}

The raw data, the information extracted by the signal processing and the analysis results are stored in a hierarchical structure. This approach aims to increase flexibility and enables a multi-user 


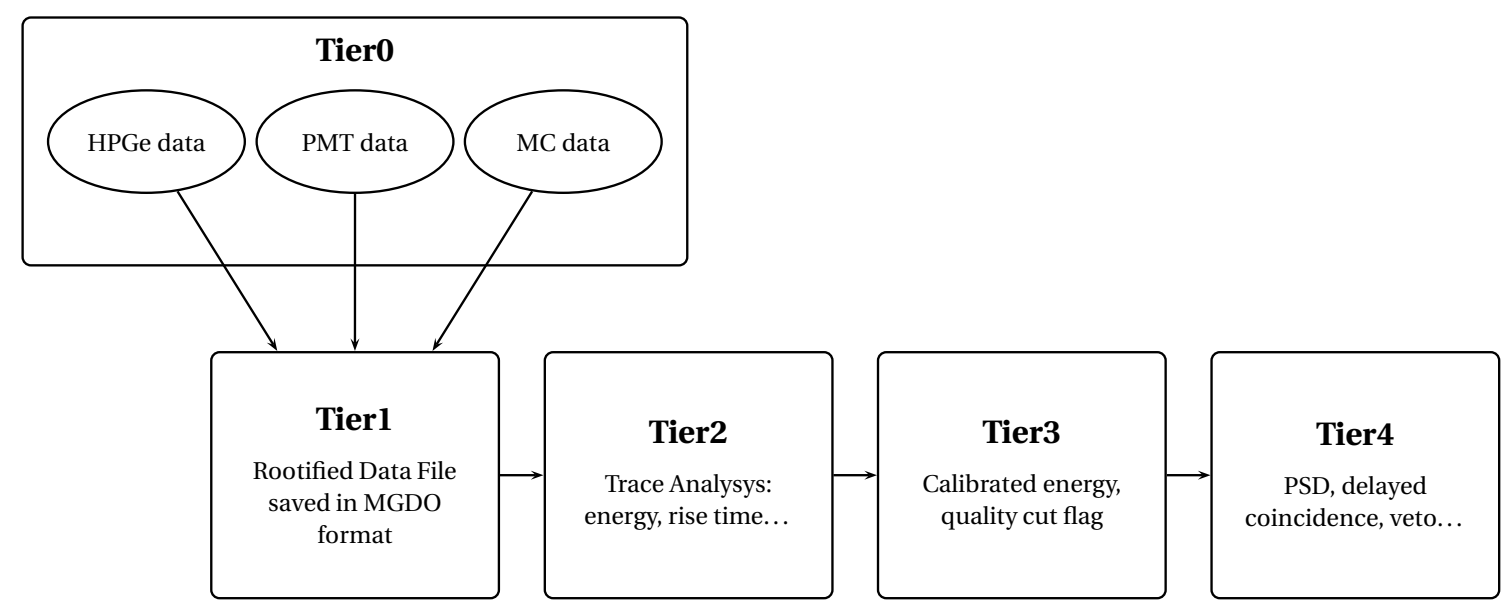

Figure 1. The hierarchical organization of the data in GELATIO. The framework organizes the output of each step of the analysis in a different level (Tier) starting from the raw data (Tier0) up to the condensed parameter of the final analysis. The Tier1 contains the same information of the raw data but encoded with a different format based on Root [1] and MGDO. More details can be found in sect. 3.2.

customized data analysis. Alternative analyses can be created as forks of the default one, sharing part of the data flow until a given level and then creating a parallel stream of information. The multi-level structure includes naturally in the framework the conversion of the raw data into a new standardized format which is optimized for signal processing and data storage. After the conversion, all data can be processed along the same analysis stream independently of the parent data acquisition (DAQ) system data format, including data produced by Monte Carlo simulations.

The multi-tier structure is depicted in Figure 1]. The raw data provided by the different DAQ systems and by the Monte Carlo simulations are stored in the lowest level ("Tier0"). Data are then converted into a new encoding and stored as "Tier1". The first two tiers contain exactly the same amount of information, the only difference being that while the Tier0 is the native DAQ format, the Tier1 is a standardized format that can be chosen to be solid, flexible, exportable and easily readable. The Tier1 data are distributed to the GERDA collaborators as the starting point for the analysis. Higher-level tiers - which are produced from the Tier1 - are meant to contain the analysis results. The "Tier2" files store the output information obtained by applying the digital analysis to the individual traces of each event, as rise time, amplitude, average noise, baseline average value, etc. Similarly, the "Tier3" files store information extracted from the Tier2, e.g. the actual energy spectrum obtained by calibrating the amplitude spectrum with the appropriate calibration curves. As the analysis becomes more and more refined (noise rejection, pulse shape discrimination analysis, delayed coincidence, veto, etc.), the information can be stored in higher-level tiers.

A drawback of this approach is the additional request for disk space due to the coexistence of the same information in both the Tier0 and the Tier1. On the other hand the raw data are not meant to be distributed because the Collaboration plans to blind the events with energy close to the region of interest $\left(Q_{\beta \beta}\right.$-value of $\left.{ }^{76} \mathrm{Ge}\right)$. Raw data will be backed up in the computing centers of three different institutions and used only to generate the Tier1. In the conversion process the data blinding is applied and only the resulting Tier1 data are released to the Collaboration. 


\subsection{Modular digital signal processing}

The core of GELATIO is the digital signal processing which creates the Tier2 files starting from the detector signals stored in the Tier1. In this step different algorithms are applied to the signals in order to extract efficiently the pulse shape information, for instance maximum amplitude, rise time, baseline slope, etc. In $\gamma$-ray spectroscopy these operations are usually performed by chains of elementary digital filters (differentiation, integration, deconvolution, etc.) optimized to reduce the noise and to extract the information with high precision.

To support a highly customizable analysis, the design of GELATIO is based on a modular approach. The analysis is divided into modules, each handling a unique and consistent task of the digital data processing, as for instance energy reconstruction and baseline subtraction. Each module includes a chain of elementary digital filters which is optimized to extract the information of interest from the signal trace. The computed information as well as the shaped traces can be used as input for other modules. The list of active modules and the parameters used by the internal chain of filters are controlled by the end user through an appropriate ASCII initialization file (INI file).

This design provides a wide flexibility as complicated chains of modules can be created by the user in an open and transparent way through the INI file. The same module can be run many times within the same execution and used in different chains, each time with different sets of parameters. Moreover, the user can easily create new modules implementing his own customized analysis tasks. The new modules are immediately available for registration in the INI file and can be combined with the standard ones to create new chains. The data flow and the INI file of an illustrative analysis are reported in Figure 2 and Figure 3, respectively. This solution enhances also the re-use of the analysis algorithms and avoids code proliferation.

\section{Implementation}

The core of the framework is implemented in $\mathrm{C}++$ to ensure an easy and natural interfacing with several scientific general-purpose projects. This choice provides also high computational performances, wide flexibility thanks to the object-oriented programming support, and cross-platform compatibility. GELATIO depends on the CLHEP [13] and FFTW3 [14] libraries for scientific computing, and on the Rоот [11] and ТАм [15] libraries for the management of the modular analysis, the data storage and the graphical tools. All the external software packages mentioned above are freeware and open-source. GELATIO additionally depends on the MGDO package for the basic digital signal processing algorithms and for the definition of the objects used to encapsulate the information in the Tier1 output. MGDO (Majorana-GERDA Data Objects) is a set of libraries that are jointly maintained and developed by the Majorana [16] and GERDA collaborations. They are specifically designed to improve the encapsulation and the handling of complex data as dedicated $\mathrm{C}++$ objects.

GELATIO is distributed to the GERDA collaborators in the form of a source code. It can be compiled on any platform supporting GNU C++, including Linux and MacOS. A configure script takes care of setting automatically the appropriate paths and environment variables necessary to compile the code. The installation procedure has been successfully tested on both 32- and 64-bit operating systems. 


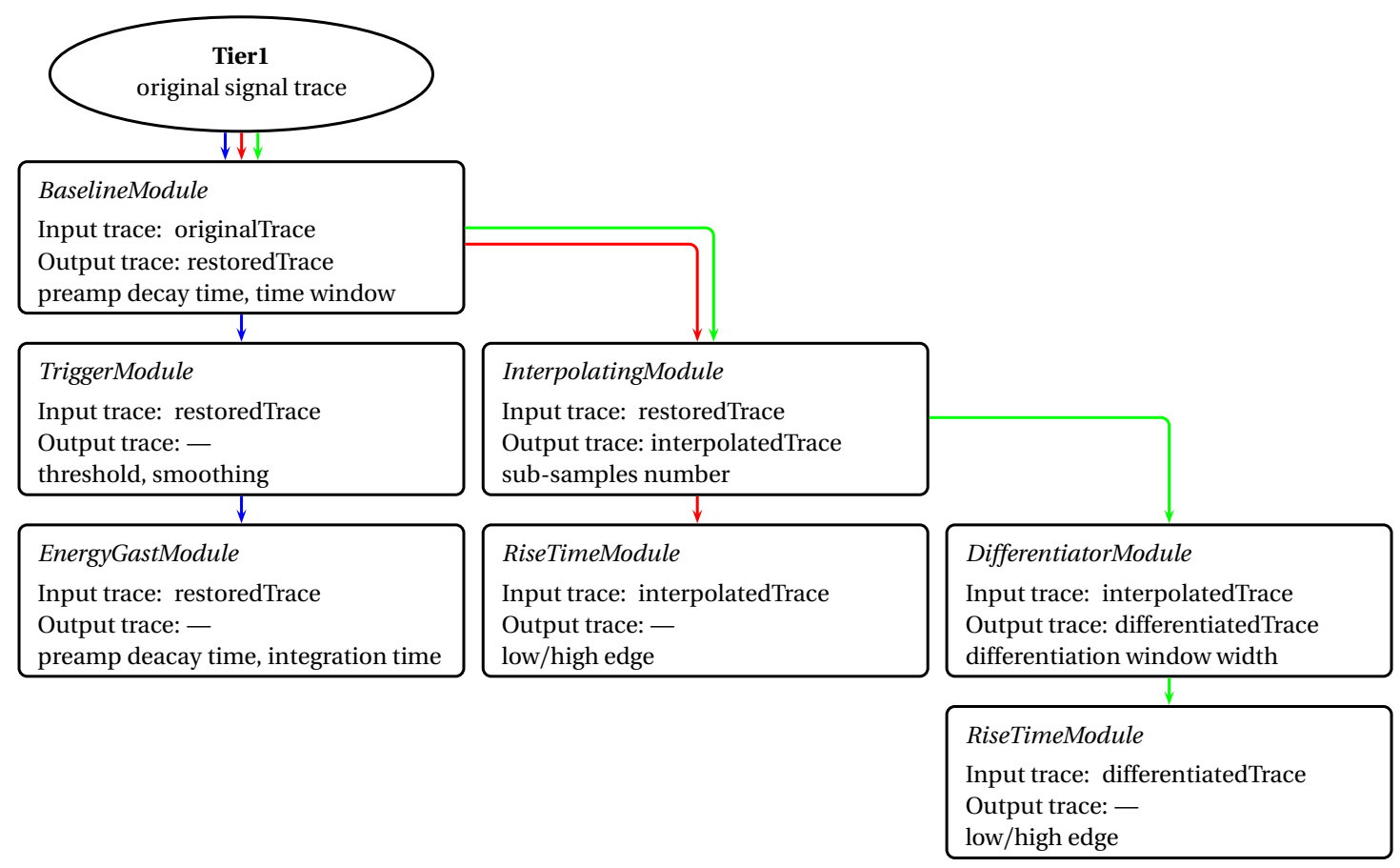

Figure 2. Data flow of an illustrative analysis which uses three chains of modules. The first chain (blue arrows) reconstructs the event amplitude and includes the baseline restoration and correction for pile-up (BaselineModule) and the computation of the trigger time (TriggerModule). The signal shaped by BaselineModule and the trigger computed by TriggerModule are used as input for EnergyGastModule, which reconstructs the pulse amplitude according to the Gast moving-window-deconvolution approach [12]. The second chain (red arrows) is used to estimate the rise time of the signal. The traces are first shaped by BaselineModule, interpolated by InterpolatingModule to push the time resolution below the sampling frequency and finally processed by RiseTimeModule. The last chain (green arrows) computes the rise time of the derivative of the signals (current signal) and shares the first three modules with the previous chain. The signal shaped by InterpolatingModule is fed to DifferentiatorModule to compute the numerical derivative, and eventually is parsed to a second instance of RiseTimeModule. The figure shows for each module the input and output trace (second and third line) and the main parameters used by the internal algorithms (last line).

To ensure flexibility and good computational performances, the framework includes both compiled and interpreted code. In section 3.1 and section 3.2 the implementation of the two executables in charge for the raw data to Tier1 conversion and for the actual modular data analysis (Tier2 production) are described in detail. Then the suite of Bash and Python scripts to handle the data streaming through the different tiers is presented in section 3.3. Section 3.4 eventually describes the graphical interface used to display the event traces, define the shaping parameters and create the INI files.

\subsection{Conversion of raw data to the analysis format}

The binary data format chosen for Tier1 is a ROOT file containing a TTree of MGDO objects (MGTEvent and MGTRun). The MGDO objects employed in the Tier1 output are containers 
Such an approach makes the framework able to handle in a completely transparent way a data stream containing an unspecified number of DAQ channels, each with digitized traces of unspecified length. This is required because the number of operational detectors and the digitization parameters will change during the experiment lifetime. Similarly, GELATIO is able to handle a mixed stream coming from different types of detectors (e.g. HPGe detectors and PMTs in LARGE). The Tier1 data format is used also as output of the pulse simulation software developed in the framework of GERDA [7]. Consequently, the simulated traces can be treated in the same way of the experimental data and be processed along the same analysis flow, enabling an easy and direct Monte Carlo-to-data comparison.

The testing and benchmarking of GELATIO was performed by using the main GERDA server. The server runs Scientific Linux 5.5 64-bit and mounts a Dual Xeon E5620 CPU $(2 \times 4$ cores at $2.4 \mathrm{GHz}$ with $2 \times 12 \mathrm{Mb}$ cache), $16 \mathrm{~GB}$ of RAM, and 20 hard-disks ( $2 \mathrm{~TB}$ ) connected through a SATA $3 \mathrm{~Gb} / \mathrm{s}$ interface and operated in RAID6/XFS. The computational performances of the conversion program are affected by the encoding and type of raw data and by the ROOT compression options required. For instance, a typical GERDA calibration run (about 3.5 $10^{6}$ waveforms, each having 4096 samples, total size about $30 \mathrm{~GB}$ ) is completely converted into the Tier1 format by the reference machine in about 100 minutes using a single thread. The processing time is substantially reduced if the ROOT compression option is switched off, at the expense of additional disk space. It has to be emphasized that the conversion of raw data into the Tier1 format must be done only once, so the best compromise is usually to pay in CPU computing time to obtain a smaller output.

\subsection{Implementation of digital signal processing}

To implement the modular analysis following the design discussed in section 2.2, the framework relies on the Tree-Analysis Module (TAM) package. TAM [15] is a free package for RoOT developed to provide a very general and modular interface for analyzing data stored in a TTree. The software combines the features of two RоOT objects: the TSelector method for processing trees and the TTask for handling a hierarchical structure of modules in a user-transparent way.

In GELATIO each analysis module is a concrete class derived by the basic interface TAModule provided by TAM via an additional GELATIO-specific base class named GERDAModule. TAM is used to handle the event loading from the Tier1 file, the exchange of information among different modules and the object output list. Moreover, the interfacing with TAM ensures the compatibility with the ROOT extension PROOF (Parallel ROOT Facility) [17] enabling the software to run several threads in parallel.

The executable in charge of the Tier2 creation takes care to instantiate the TAM interface initializing the analysis modules according to the instruction provided through the INI file - and to store all the outputs of the same execution in a single Rоoт file. The output is a collection of RоOт objects usually containing a Trree for each module but also histograms or signal traces. The software provides also a master TTree which can be used for unrestricted and parallel access to information contained in any other Trree in the file, via the RоOт friendship mechanism.

The CPU time required to run an analysis depends on the number of active channels and modules as well as on the module parameters. For instance, the standard GERDA analysis chain includes baseline restoration, trigger position and rise time computation, and two independent modules for amplitude reconstruction. A typical calibration run containing $3.5 \cdot 10^{6}$ traces, each 4096 samples 


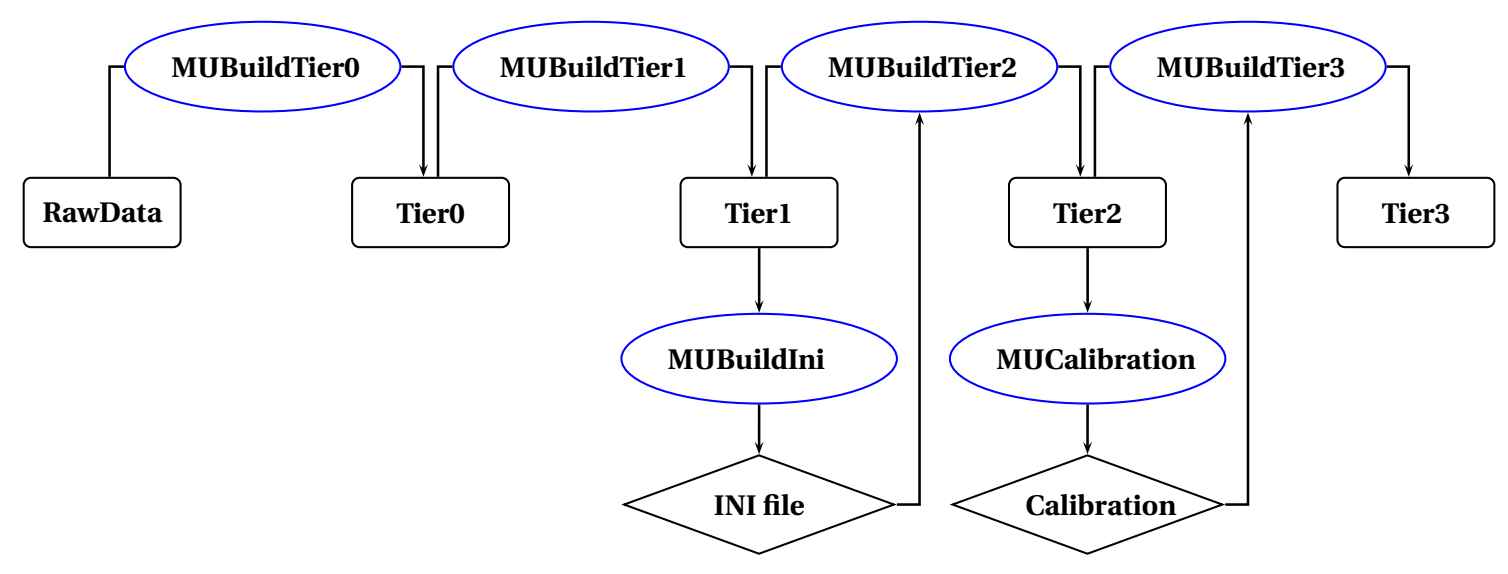

Figure 4. Data flow of the information through the different tiers performed by using the suite of utilities (blue nodes) included in GELATIO. These utilities are designed to provide an interface to import raw data in the framework, convert them into the Tier1 format, create the INI file and run the digital signal processing. Moreover, the utilities allow the user to perform an interactive and graphical calibration of the amplitude spectra.

long, is processed according to the GERDA standard analysis chain in less than 4 hours by using a single thread of the reference benchmark machine.

\subsection{Utilities}

To help the handling of the data stream through the different tiers, GELATIO includes a suite of utilities implemented as Bash and Python scripts. The utilities work over a well-defined directory structure ("analysis file system") and provide a user-interface for each step of the analysis. The scripts take care of identifying which files should be processed and of storing the results of each step in the proper directory, including a log file collecting the standard outputs generated by the executables. Since the information is stored in fixed directories inside the file system, each user is immediately able to recover any output.

In Figure 4, each step of the data flow is depicted together with the associated utility. The utilities up to the Tier2 builder are implemented as simple Bash scripts and are designed to provide an interface to the file system and to run the GELATIO executables with the proper options. The last two routines are more complicated as they are supposed to provide to the user an interactive graphical tool to calibrate the energy spectra and to create the Tier3. Moreover, they take care of storing the calibrating parameters of each channel in different directories of the output TFile, together with all the information important for the debugging, i.e. the calibration log files and the plots of the fits. These utilities are implemented in Python to take advantage of the Rоot binding PyROOT which enables cross-calls from Python to ROOT/CINT [11].

\subsection{The graphical interface}

The graphical user interface (GUI) integrated in GELATIO (Figure 5) is a general and powerful tool developed to create and handle INI files. The interface is implemented entirely by using RoOT 


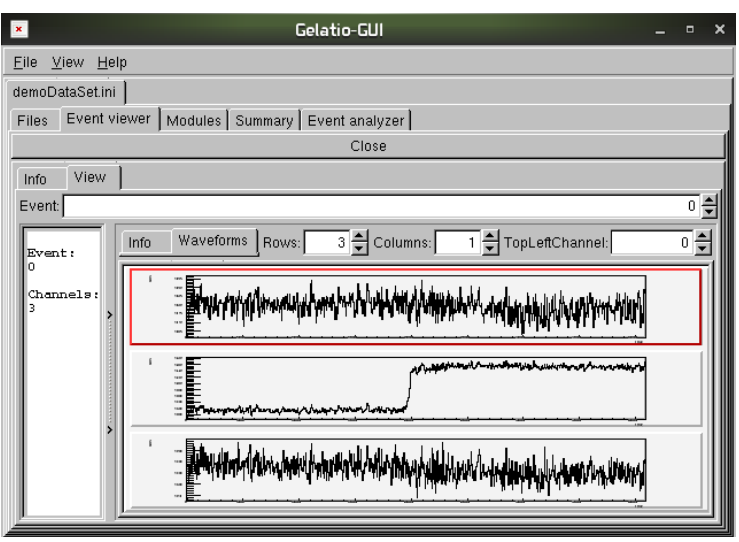

(a)

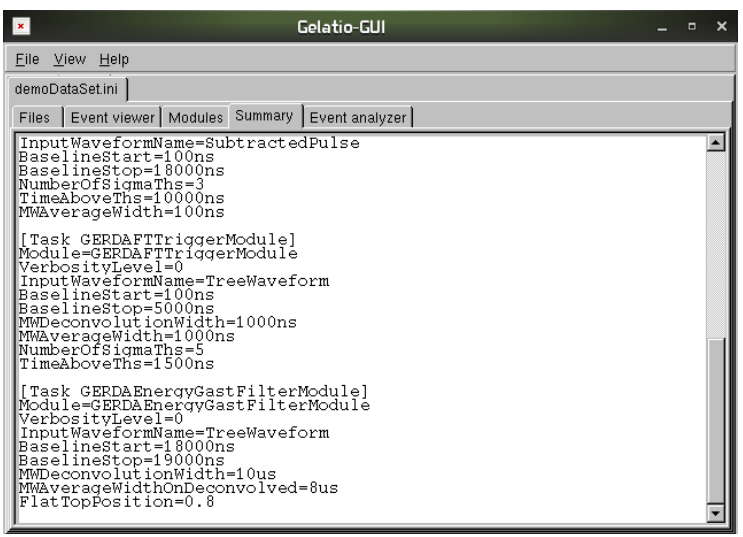

(c)

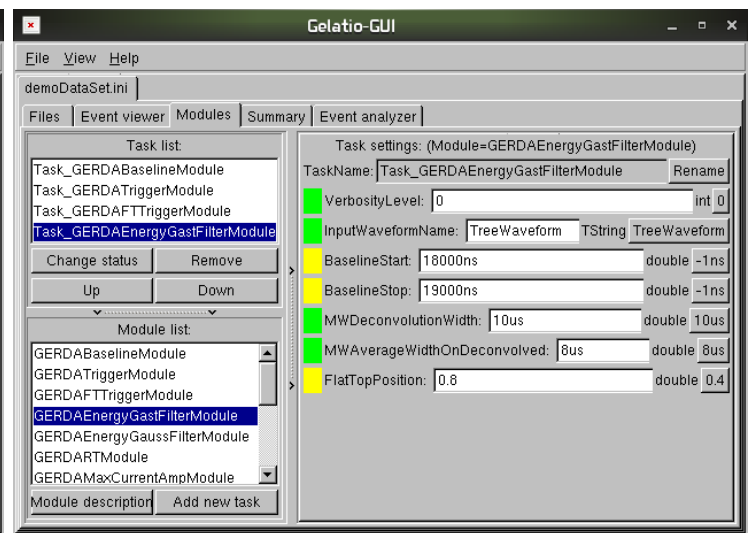

(b)

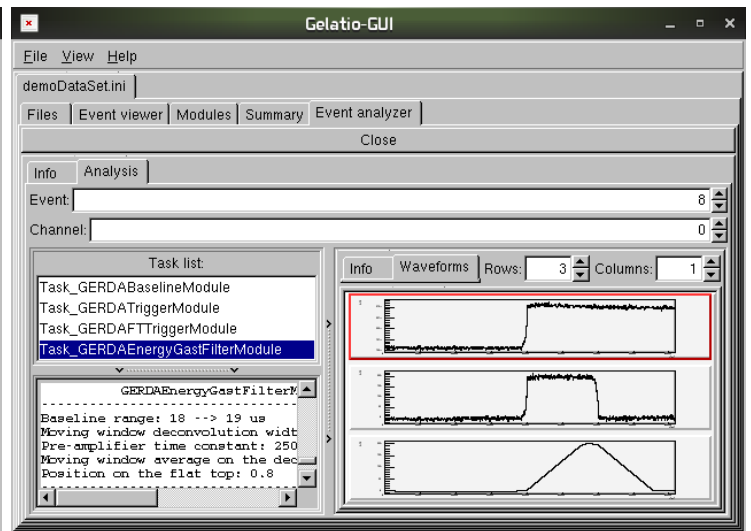

(d)

Figure 5. Screen shots of the GELATIO GUI. The input Tier1 file comes from a GERDA background run and contains three traces per event. The screen shots show the tools and utilities available in the GUI: (a) Event displayer. The signals from the three channels are displayed together. (b) INI file editor. It can be used to select and customize the analysis tasks to be performed. The "Module list" contains all the analysis modules available in GELATIO. (c) INI output summary. It shows the human-readable INI file produced according to the user choices in the INI file editor. (d) Event analyzer. To apply the full analysis chain to a given trace. The screen shot shows the intermediate shaped traces calculated by the analysis module which implements the Gast [12] algorithm for amplitude reconstruction.

graphical components. Despite the lack of flexibility and the intrinsic limitations of the ROOT graphical libraries, the native ROOT solution has been preferred to ensure smooth integration with the rest of the framework and to minimize external dependencies.

The GUI aims to help the user in the creation and in the testing of the INI files and is able to handle multiple files at the same time. The layout is based on five main tabs:

- Files: to select the Tier1 files to analyze. The files can be selected by using a graphical window, and multiple files can be selected at the same time.

- Event viewer (Figure 5.a): to browse channel-by-channel the events contained in the selected Tier1 files. Several channels can be displayed at the same time. 
- Modules (Figure 5.b): to configure the tasks to be used for the analysis. A "task" is a module with a particular set of parameters. The modules to be activated can be selected from a list. For each module the GUI displays the full list of the customizable parameters which can be edited interactively by the user. Since modules can be possibly configured with many independent parameters, it is particularly useful to have a visual list of them and of their default values. For each parameter the GUI shows a color-code label. The green code means that the selected value is equal to the default; the yellow code stands for a valid value which is different from the default; the red code indicates an invalid parameter value (wrong unit, for instance).

- Summary (Figure 5.c): to view the resulting INI file.

- Event Analyzer (Figure 5.d): to test the INI file, namely the analysis chain, on a single event in the Tier1 file. It is possible to display the input/output traces of each module and also the intermediate traces produced along the digital processing. Such a tool proved to be very useful for debugging the analysis chain and for tuning/optimizing the values of the parameters to be used for a given data set.

\section{Application and Benchmarking}

The framework has been used up to now to handle and analyze data from several GERDA-related activities, including calibrations with radioactive sources. In particular, GELATIO was used for the data analysis of the GERDA R\&D activities related to the Broad Energy Germanium (BEGe) detectors. The experimental data [18, [19, 20] and the corresponding Monte Carlo simulations [[] were processed on the same footing to compare directly the results. The pulse shape discrimination algorithms developed for the BEGe detectors [6, 21] were coded as dedicated GELATIO modules and successfully applied to data. Results in term of discrimination efficiency calculated with the Gelatio modules are consistent with those obtained with the dedicated code of Ref. [6]. The present analysis of the LARGE data [22] is based on GELATIO; in this case, the framework is able to handle the data streams coming from the HPGe detector and from the PMTs of the instrumented liquid argon veto. Finally, the framework is currently used for the reference data analysis of the data collected in the GERDA commissioning with three HPGe detectors.

Up to now GELATIO was used on data files coming from six independent DAQ systems, differing for binary data format, number of channels, sampling frequency and sampling window. It proved to be able to handle correctly multiple DAQ channels - possibly referred to different kinds of detectors - and pile-up corrections that must be applied for source calibration runs, because of the higher counting rate. The software turned out to be stable and robust. Fixes for the few minor bugs reported since the release of the stable version are made available in regularly-updated versions of GELATIO.

Figure 6 shows the distribution of the trace trigger time (upper panel) and the amplitude spectrum (lower panel) of one of the HPGe detectors deployed in GERDA irradiated with a ${ }^{228} \mathrm{Th}$ calibration source. The distributions are obtained at the end of the Gelatio-based analysis flow, using the reference INI file described in section 3.2. The amplitude spectrum of Figure 6 has been produced after having discarded those events having a reconstructed trigger time significantly far 

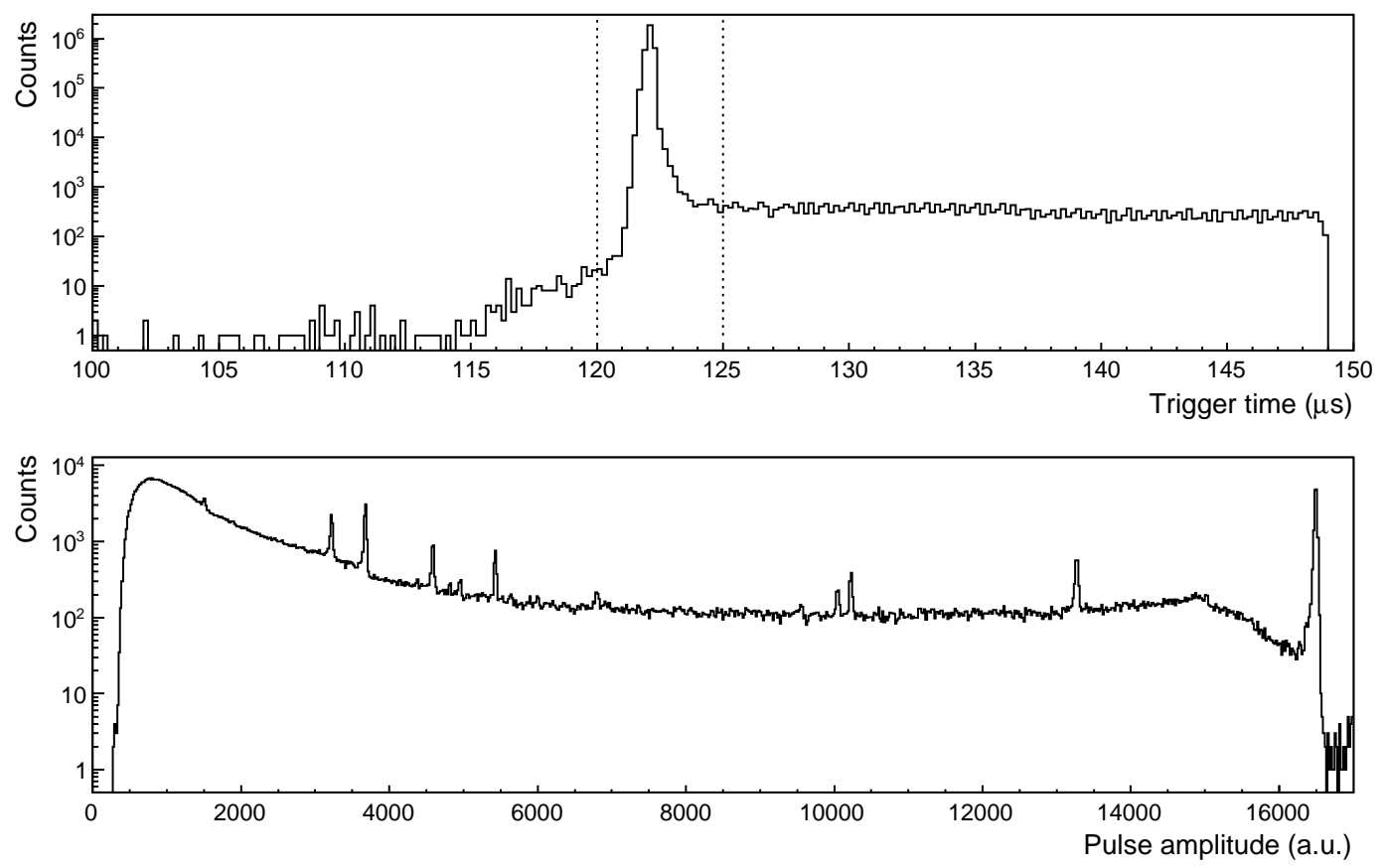

Figure 6. Analysis results obtained by the GELATIO processing of a ${ }^{228} \mathrm{Th}$ calibration taken in GERDA. Upper panel: distribution of the reconstructed trigger time for one of the three detectors. The DAQ chain is set in order to have the trace at about $120 \mu \mathrm{s}$ after the start of the sampling window. Lower panel: amplitude distribution obtained for the same data set from the Gaussian shaping algorithm. The amplitude spectrum includes only the signals having the trigger time between $120 \mu \mathrm{s}$ and $125 \mu \mathrm{s}$ (see dashed lines in the upper panel) in order to discard accidental and mis-reconstructed events. The management of the GELATIO output is performed via the master TTree in the Tier2 file.

from the expected value. Notice that trigger times and trace amplitudes are calculated by two independent analysis modules and stored in two separate TTrees in the Tier2 file. The amplitude and trigger information from the different TTrees is correlated via the master TTree created by Gelatio.

The amplitude was reconstructed off-line using two independent algorithms - Gaussian shaping (shown in Figure (6) and Gast method [12] - both implemented as GELATIO modules. After the appropriate tuning of the parameters, the two methods give equivalent results for the energy resolution. The energy reconstruction and resolution provided by GELATIO for the GERDA data were compared to the results obtained with an independent and dedicated analysis code and found to be consistent.

\section{Conclusions}

A powerful and flexible software framework called GELATIO has been developed to handle the full analysis flow of the GERDA experiment and the related R\&D activities. The software is written in $\mathrm{C}++$ and is based on an object oriented design. GELATIO contains executable programs and utility scripts which take care of the full analysis chain, starting from the raw data up to the final condensed 
parameters. Complex analysis, possibly involving energy reconstruction, detector coincidence and pulse shape discrimination, can be hence managed in a very transparent and general way.

As raw data are converted in a standardized RоOT-based format, data streams originated by the different DAQ systems used in the GERDA and by Monte Carlo simulations can be treated with the same algorithms and along the same analysis flow, easing the cross-check and inter-comparison among the results.

A modular analysis approach based on TAM is used, designed to sub-divide the analysis work in many nearly-independent tasks that can be activated interactively and possibly run in parallel in multi-thread systems. The active modules and the corresponding parameters are selected by the end user via a human-readable INI file or a graphical interface. The GUI acts also by event displayer and by interactive analysis manager, able to show each intermediate step of the modular analysis.

A stable version of Gelatio is presently available for the Gerda Collaboration and the framework has been widely used for the data analysis in the GERDA commissioning. The GERDA database system, which is currently under development, supports GELATIO in input and output. It is able to import the analysis results from Tier2 files and to generate Tier1 files from a custom selection of events made through SQL [23] queries. GELATIO is also used in other GERDA-related activities, including LARGE and the characterization of prototype BEGe detectors.

The software has been validated against other independent and dedicated analysis codes. Furthermore, GELATIO proved to be robust, effective and flexible enough to handle a complex analysis stream from a real-life multi-channel experiment. GELATIO could be used in any experimental activities involving digital pulse shape analysis of HPGe detector signals.

\section{Acknowledgments}

We would like to acknowledge our colleagues of the GERDA Collaboration, especially B. Schwingenheuer, for many invaluable advices concerning analysis algorithms and data analysis, as well as for providing results from his own analysis code that were used to benchmark GELATIO. We want also to thank D. Bazzacco and C. A. Ur for all the stimulating discussions concerning $\gamma$-ray digital filters and the BEGe team for having been our beta tester and having provided very valuable feedback, in particular D. Budjáš and A. Lubashevskiy.

We express our gratitude to the colleagues from the Majorana Collaboration, and specifically J. Detwiler and M. Marino, for pointing out to us the TAM package and for many suggestions about software design and implementation. Furthermore, we would like to thank S. Stalio from the LNGS IT Service for help and support with the LNGS computing cluster.

This work was supported in part by the Transregio Sonderforschungsbereich SFB/TR27 "Neutrinos and Beyond" by the Deutsche Forschungsgemeinschaft and by the Munich Cluster of Excellence "Origin and Structure of the Universe".

\section{References}

[1] GERDA Collaboration, I. Abt et al., GERDA: The GERmanium Detector Array for the search of neutrinoless $\beta \beta$ decay of ${ }^{76}$ Ge at LNGS, Proposal, http://www.mpi-hd.mpg.de/ge76.

[2] GERDA Collaboration, S. Schönert et al., The GERmanium Detector Array (GERDA) for the search of neutrinoless $\beta \beta$ decays of ${ }^{76}$ Ge at LNGS, Nucl. Phys. B, Proc. Suppl. 145 (2005) 242. 
[3] M. Gunther et al., Heidelberg - Moscow beta beta experiment with Ge-76: Full setup with five detectors, Phys. Rev. D 55 (1997) 54.

[4] H.V. Klapdor-Kleingrothaus, I.V. Krivosheina, A. Dietz, O. Chkvorets, Search for neutrinoless double beta decay with enriched ${ }^{76}$ Ge in Gran Sasso 1990-2003, Phys. Lett. B 586 (2004) 198.

[5] C.E. Aalseth et al., IGEX ${ }^{76}$ Ge neutrinoless double-beta decay experiment: Prospects for next generation experiments, Phys. Rev. D 65 (2002) 092007.

[6] D. Budjáš, M. Barnabé Heider, O. Chkvorets, N. Khanbekov and S. Schönert, Pulse shape discrimination studies with a Broad-Energy Germanium detector for signal identification and background suppression in the GERDA double beta decay experiment, JINST 4 (2009) P10007.

[7] M. Agostini et al., Signal modeling of HPGe detectors with a small read-out electrode and application to neutrinoless double beta decay search in Ge-76, JINST 6 (2011) P03005.

[8] D. Gonzalez et al., Pulse shape discrimination in the IGEX experiment, Nucl. Instrum. Meth. A 515 (2003) 634 [hep-ex/0302018].

[9] J. Hellmig, H. V. Klapdor-Kleingrothaus, Identification of single-site events in germanium detectors by digital pulse shape analysis, Nucl. Instrum. Meth. A 455 (2000) 638.

[10] M. Di Marco, P. Peiffer, S. .Schonert, LArGe: Background suppression using liquid argon (LAr) scintillation for 0 nu beta beta decay search with enriched germanium (Ge) detectors, Nucl. Phys. Proc. Suppl. 172 (2007) 45 [physics/0701001].

[11] R. Brun and F. Rademakers, ROOT - An Object Oriented Data Analysis Framework, Nucl. Instrum. Meth. A 389 (1997) 81; URL http://root.cern.ch/.

[12] J. Stein, F. Scheuer, W. Gast, A. Georgiev, X-ray detectors with digitalizer preamplifiers, Nucl. Instrum. Meth. B 113 (1996) 141.

[13] L. Lönnblad, CLHEP: a project for designing a C++ class library for high energy physics, Comput. Phys. Commun. 84 (1994) 307; URL http://proj-clhep.web.cern.ch.

[14] M. Frigo and S.G. Johnson, The Design and Implementation of FFTW3, Proceedings of the IEEE 93 (2005), 216; URL http://www.fftw.org/.

[15] M. Ballintijn, C. Loizides and C. Reed, Tree Analysis Modules, URL http://www.cmsaf.mit.edu/twiki/bin/view/Software/TAM.

[16] C. E. Aalseth et al. [ Majorana Collaboration ], The Majorana neutrinoless double beta decay experiment, Phys. Atom. Nucl. 67 (2004) 2002 [hep-ex/0405008].

[17] M. Ballintijn, R. Brun, F. Rademakers, G. Roland, The PROOF Distributed Parallel Analysis Framework based on ROOT, [arxiv:physics/0306110v1]; URL http://root.cern.ch/drupal/content/proof.

[18] M. Agostini et al., Characterization of a broad energy germanium detector and application to neutrinoless double beta decay search in ${ }^{76}$ Ge, JINST 6 (2011) P04006.

[19] M. Agostini et al., Procurement, production and testing of BEGe detectors in ${ }^{76} G e$, to appear in Nucl. Phys. B, Proc. Suppl. (Neutrino 2010).

[20] A. di Vacri et al., Characterization of Broad Energy Germanium Detector (BEGe) as a candidate for the GERDA experiment, IEEE Nucl. Sci. Symp., Conf. Record 2009 (2009) 1761.

[21] D. Budjáš, Germanium detector studies in the framework of the GERDA experiment, Ph. D. thesis, University of Heidelberg (2009). 
[22] M. Heisel, $L A r G e-A$ liquid argon scintillation veto for GERDA, Ph. D. thesis, University of Heidelberg (2011).

[23] SQL - Structured Query Language - Standard ISO/IEC 9075. 